\title{
Practical Collisions for SHAMATA-256
}

\author{
Sebastiaan Indesteege $e^{1,2, \star}$, Florian Mendel $^{3}$, Bart Preneel ${ }^{1,2}$, \\ and Martin Schläffer ${ }^{3}$
}

1 Department of Electrical Engineering ESAT/COSIC, Katholieke Universiteit

Leuven. Kasteelpark Arenberg 10, B-3001 Heverlee, Belgium

2 Interdisciplinary Institute for BroadBand Technology (IBBT), Belgium

3 Institute for Applied Information Processing and Communications

Inffeldgasse 16a, A-8010 Graz, Austria

\begin{abstract}
In this paper, we present a collision attack on the SHA-3 submission SHAMATA. SHAMATA is a stream cipher-like hash function design with components of the AES, and it is one of the fastest submitted hash functions. In our attack, we show weaknesses in the message injection and state update of SHAMATA. It is possible to find certain message differences that do not get changed by the message expansion and non-linear part of the state update function. This allows us to find a differential path with a complexity of about $2^{96}$ for SHAMATA-256 and about $2^{110}$ for SHAMATA-512, using a linear low-weight codeword search. Using an efficient guess-and-determine technique we can significantly improve the complexity of this differential path for SHAMATA256. With a complexity of about $2^{40}$ we are even able to construct practical collisions for the full hash function SHAMATA-256.
\end{abstract}

Keywords: SHAMATA, SHA-3 candidate, hash function, collision attack.

\section{Introduction}

A cryptographic hash function $H$ maps a message $M$ of arbitrary length to a fixed-length hash value $h$. Informally, a cryptographic hash function has to fulfil the following security requirements:

- Collision resistance: it is infeasible to find two messages $M$ and $M^{*}$, with $M^{*} \neq M$, such that $H(M)=H\left(M^{*}\right)$.

- Second preimage resistance: for a given message $M$, it is infeasible to find a second message $M^{*} \neq M$ such that $H(M)=H\left(M^{*}\right)$.

- Preimage resistance: for a given hash value $h$, it is infeasible to find a message $M$ such that $H(M)=h$.

The resistance of a hash function to collision and (second) preimage attacks depends in the first place on the length $n$ of the hash value. Regardless of how a hash function is designed, an adversary will always be able to find preimages or

\footnotetext{
^ F.W.O. Research Assistant, Fund for Scientific Research — Flanders (Belgium).
} 
second preimages after trying out about $2^{n}$ different messages. Finding collisions requires a much smaller number of trials. Due to the birthday paradox, collisions can be found in a generic way with an effort of only about $2^{n / 2}$. A hash function is said to achieve ideal security if these bounds are guaranteed.

In the last few years, the cryptanalysis of hash functions has become an important topic within the cryptographic community. Especially the collision attacks on the MD4 family of hash functions (MD5, SHA-1) have diminished the confidence in the security of these commonly used hash functions. Therefore, NIST has started the SHA-3 competition [7] to find a successor for the SHA-1 and SHA-2 hash functions. The goal is to find a hash function which is fast and still secure within the next few decades.

Many new and interesting hash functions have been proposed. One of them is SHAMATA [1]. Out of the 51 first round candidates, SHAMATA is one of the fastest submissions having a speed of 8-11 cycles/byte on 64-bit and 15-22 cycles/byte on 32-bit platforms [1. It is a register based design, similar to the hash function PANAMA [5] and also bears resemblance to the sponge construction [2].

In this work, we analyse the security of the hash function SHAMATA. After a description of SHAMATA in Sect. 2, we analyse some basic differential properties of the message injection and state update function in Sect. 3. We show how to efficiently linearise SHAMATA by considering special XOR differences with an equal difference in all bytes. In Sect. 4, we construct a good differential path for the linearised variant of SHAMATA using a low-weight codeword search. Section 5 explains how basic message modification techniques allows us to construct a collision attack with a complexity of $2^{96}$ for SHAMATA-256 and $2^{110}$ for SHAMATA-512, based on this differential path. For SHAMATA-256, the attack is improved further to a complexity of only $2^{40}$ SHAMATA rounds using a complex guess-and-determine strategy. This attack is practical, and we show a collision example in App. A. We conclude our analysis of the hash function SHAMATA in Sect. 6.

\section{Description of SHAMATA}

In this section, we give a brief description of the hash function SHAMATA. SHAMATA is a register based hash function design that operates on an internal state of 2048 bits and produces a hash value of $224,256,384$ or 512 bits. The internal state consists of two parts: the main mixing register $B_{3}, \ldots, B_{0}$ and the second mixing register $K_{11}, \ldots, K_{0}$. Internally, SHAMATA uses rounds of the AES block cipher [6] as building blocks.

First, the message is padded to an integer number of 128-bit blocks using classical Merkle-Damgård strengthening, like in the MD4 family. The registers comprising the internal state of SHAMATA are set to their initial values, which depend on the digest length used. Then, each 128-bit message block is used once to update the internal state as described below. Finally, the finalisation phase of SHAMATA generates the output digest from the internal state. For a detailed description of the initialisation and finalisation phases of SHAMATA, we refer to [1, as these details are not relevant to our analysis. 


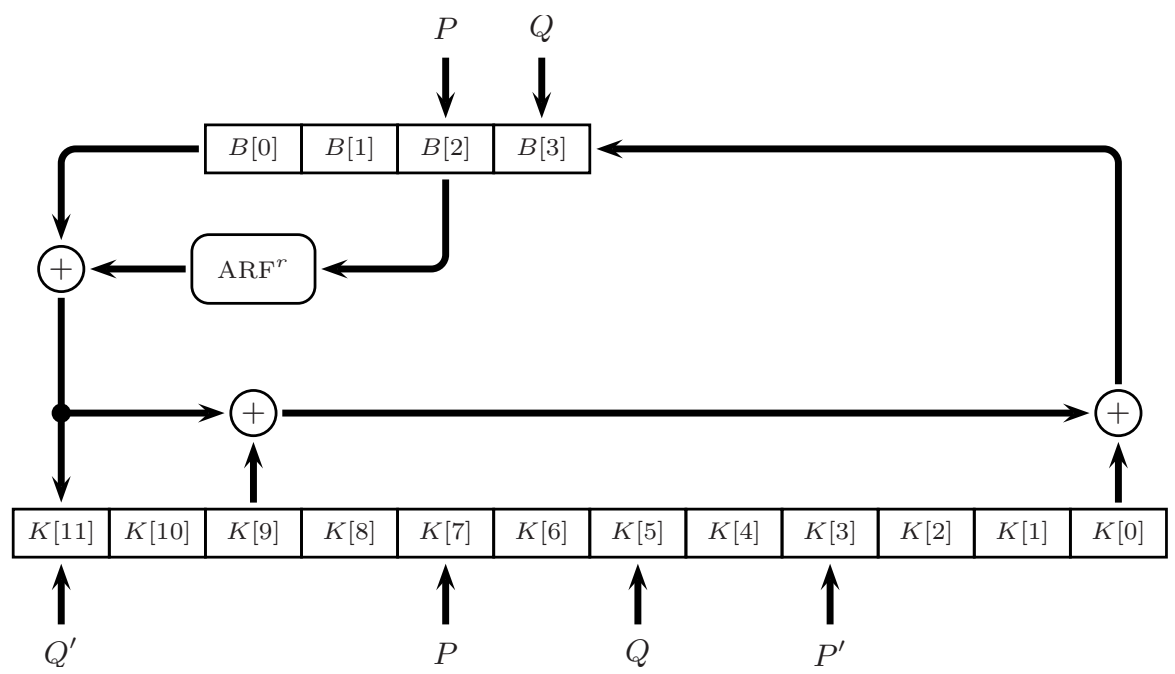

Fig. 1. The state update function of SHAMATA

\subsection{The Message Injection}

The message injection of SHAMATA updates the internal state using a 128-bit message block. The message block $M$ is first expanded as follows:

$$
\begin{array}{ll}
P=M C\left(M^{\mathrm{T}}\right), & Q=M C(M), \\
P^{\prime}=P(1) \| Q(0), & Q^{\prime}=Q(1) \| P(0) .
\end{array}
$$

Here, $M C$ is the MixColumns operation from the AES block cipher [6] and $M^{\mathrm{T}}$ is the transpose of $M$, where $M$ is viewed as a $4 \times 4$ matrix of bytes. The notation $P(i)$ denotes the $i$-th most significant 64 -bit half of the 128 -bit word $P$. Thus, $P^{\prime}$ and $Q^{\prime}$ are simply recombinations of the columns of $P$ and $Q$. These expanded message words and a block counter blockno are then added to six words of the internal state using XOR:

$$
\begin{array}{ll}
B_{2} \leftarrow B_{2} \oplus P \oplus \text { blockno }, & B_{3} \leftarrow B_{3} \oplus Q \oplus \text { blockno }, \\
K_{3} \leftarrow K_{3} \oplus P^{\prime}, & K_{5} \leftarrow K_{5} \oplus Q, \\
K_{7} \leftarrow K_{7} \oplus P, & K_{11} \leftarrow K_{11} \oplus Q^{\prime} .
\end{array}
$$

\subsection{The State Update Function}

After the expanded message words have been added, the state update function updates the internal state by clocking the registers of the internal state twice, as is shown in Fig. 1] Formally, these two clockings can be written as 


$$
\begin{aligned}
& \text { feed } K_{1}=A R F^{r}\left(B_{2}\right) \oplus B_{0}, \quad \text { feed } B_{1}=\text { feed } K_{1} \oplus K_{9} \oplus K_{0} \quad, \\
& \text { feed } K_{2}=A R F^{r}\left(B_{3}\right) \oplus B_{1}, \quad \text { feed } B_{2}=\text { feed } K_{2} \oplus K_{10} \oplus K_{1} \text {, } \\
& B_{i} \leftarrow B_{i+2} \text { for } i=0,1, \quad K_{i} \leftarrow K_{i+2} \text { for } i=0, \ldots, 9 \text {, } \\
& B_{2} \leftarrow \text { feed } B_{1}, \quad K_{10} \leftarrow \text { feed } K_{1}, \\
& B_{3} \leftarrow \text { feed } B_{2}, \quad K_{11} \leftarrow \text { feed } K_{2} .
\end{aligned}
$$

The function $A R F^{r}$ consists of $r$ rounds of the AES block cipher [6], omitting subkey additions. Thus, the ARF function consists of the SubBytes, ShiftRows and MixColumns operations:

$$
A R F(X)=M C(S R(S B(X))) .
$$

For SHAMATA-224 and SHAMATA-256, the number of rounds $r$ is equal to one. For SHAMATA-384 and SHAMATA-512, $r$ is two.

\section{Basic Attack Strategy}

In this section, we describe the basic attack strategy to construct collisions for SHAMATA. The attack is similar to the attack on PANAMA [4]10, since we construct a collision in the internal state during the message injection phase. In this phase, the message input can be used to control the differences in the internal state. However, since the expanded message block is inserted several times into the internal state, finding a differential trail seems to be difficult at first. However, by exploiting some differential properties of the state update, we can find a differential trail for SHAMATA which results in a collision with a good probability.

\subsection{Overview of the Attack}

The main idea of the attack on SHAMATA is to insert special message differences $\Delta$, which do not get changed by the message expansion and the non-linear function $A R F^{r}$. Then, the same difference $\Delta$ will be added to six positions of the internal state by the message injection. By imposing conditions on the input of $A R F^{r}$, we can ensure that the difference $\Delta$ does not get changed by this non-linear function. Hence, all parts of the state update are linear regarding the XOR difference $\Delta$ and we can search for a differential path using basic linear algebra.

\subsection{Choosing the Message Difference}

In the message expansion of SHAMATA, the 128-bit message word $M$ is first arranged in a $4 \times 4$ array of bytes. Then, the MixColumns transformation is applied to both $M$ and $M^{T}$ and some columns are rearranged to get the expanded message blocks $P, P^{\prime}, Q$ and $Q^{\prime}$. All transformations are applied on the byte level and we can make the following observation. 
Observation 1. A message difference $\Delta$ with equal differences in all 16 bytes, results in the same difference $\Delta$ in each of the expanded message words $P, P^{\prime}$, $Q$ and $Q^{\prime}$.

Transposition and rearranging columns does not change the value of byte differences. MixColumns applies the following linear transformation over $\operatorname{GF}\left(2^{8}\right)$ to each column [6]:

$$
\begin{aligned}
& b_{0}=2 \bullet a_{0} \oplus 3 \bullet a_{1} \oplus 1 \bullet a_{2} \oplus 1 \bullet a_{3} \\
& b_{1}=1 \bullet a_{0} \oplus 2 \bullet a_{1} \oplus 3 \bullet a_{2} \oplus 1 \bullet a_{3} \\
& b_{2}=1 \bullet a_{0} \oplus 1 \bullet a_{1} \oplus 2 \bullet a_{2} \oplus 3 \bullet a_{3} \\
& b_{3}=3 \bullet a_{0} \oplus 1 \bullet a_{1} \oplus 1 \bullet a_{2} \oplus 2 \bullet a_{3}
\end{aligned}
$$

If all input values are equal to some value $a$, we get with $2 \bullet a \oplus 3 \bullet a=1 \bullet a$ :

$$
b_{i}=2 \bullet a \oplus 3 \bullet a \oplus 1 \bullet a \oplus 1 \bullet a=1 \bullet a=a .
$$

and all output values are equal. Hence, for any message difference $\Delta$ with equal values in all bytes, the same difference $\Delta$ will be injected into the 6 state words $B_{3}, B_{2}, K_{11}, K_{7}, K_{5}$ and $K_{3}$.

\subsection{Linearising $A R F^{r}$}

The only non-linear part in SHAMATA is the modified AES-round $A R F^{r}$. The function $A R F^{r}$ behaves linearly if a given input difference $\Delta$ results in the same output difference $\Delta$. This is again possible for certain differences, by additionally imposing conditions on the input values of $A R F^{r}$ :

Observation 2. There are input differences $\Delta$ of $A R F^{r}$ with equal differences in all 16 bytes, which result in the same output difference $\Delta$ for certain conditions on the input values of $A R F^{r}$.

For example, in the case of $A R F^{1}$ (SHAMATA-256), the input difference $\Delta=$ $0 \times f f, 0 x f f, \ldots$ results in the same output difference $\Delta=0 \operatorname{xff}, 0 \operatorname{xff}, \ldots$ if all input byte values are equal to either $0 \times 7 e$ or $0 x 81$. A more careful choice of the difference in the input bytes can improve the probability that the differential through $A R F^{r}$ is followed.

For $A R F^{1}$ a careful examination of the difference distribution table (DDT) of the AES S-box reveals that the best choice is a difference of 0xc5 in each byte. Indeed, this difference passes through the S-box unchanged for input values $\{0 \mathrm{x} 00,0 \mathrm{x} 1 \mathrm{~d}, 0 \mathrm{xc} 5,0 \mathrm{xd} 8\}$ and hence, with an optimal probability of $2^{-6}$. Using this difference, there are $4^{16}$ values for the input to $A R F^{1}$ which exhibit the desired differential behaviour, corresponding to a differential probability of $2^{-96}$.

In the case of $A R F^{2}$ (SHAMATA-512), we can no longer view each S-box independently. Eliminating linear steps at the in- and output, $A R F^{2}$ reduces to SubBytes, followed by MixColumns and another SubBytes operation. Thus, each column is still independent here. We have performed an exhaustive search to find the best difference consisting of 16 equal bytes that passes through $A R F^{2}$ unchanged. The best choice is a difference of $0 \times 18$ in each byte, which passes through $A R F^{2}$ for $(22)^{4}$ values, corresponding to a differential probability of $2^{-110.16}$. 


\subsection{Basic Message Modification}

In this section, we analyse the possibilities to fulfil the conditions on the input of $A R F^{r}$. For each active $A R F^{r}$ function, the input value has to be such that the difference is passed unchanged. The probability of this event was optimised in the previous section. Note however that in each round, the expanded message word $P$ is XORed directly to $B_{2}$. Hence, if the $A R F^{r}$ function in the first clocking is active, we can simply choose $M$ such that the input to $A R F^{r}$ is $X$, which is fixed to one of the "good" values ensuring that the active $A R F^{r}$ has the required differential behaviour:

$$
M=\left(M C^{-1}(P)\right)^{T}=\left(M C^{-1}\left(B_{2} \oplus X\right)\right)^{T} .
$$

If the $A R F^{r}$ function in the second clocking of a round is active, a similar approach can be used, as the message is also XORed to $B_{3}$ via $Q$, which forms the input to $A R F^{r}$ in the second clocking:

$$
M=M C^{-1}(Q)=M C^{-1}\left(B_{3} \oplus X\right) .
$$

These basic message modification techniques do not work anymore as soon as two consecutive $A R F^{r}$ functions of a single round are active. If we get a difference $\Delta$ in both $B_{2}$ and $B_{3}$ after the message injection, we can adjust only one input of the following two $A R F^{r}$ functions. The main problem here is that we do not have enough freedom to fulfil the conditions on the message input imposed by both active $A R F^{r}$ functions. Hence, in this case, one of them has to be satisfied probabilistically. The best probability is $2^{-96}$ for $A R F^{1}$ and $2^{-110.16}$ for $A R F^{2}$, as was shown in Sect. 3.3.

Hence, we will aim for a differential path with a low number of consecutive active $A R F^{r}$ functions (see Sect. 4). Unfortunately, in any differential path, we always get a difference in both, $B_{2}$ and $B_{3}$ after the first message injection. However, in Sect. 5.2, we show how we can still fulfil both conditions for SHAMATA-256 with much less effort, such that the attack becomes practical.

\section{Finding a Good Differential Path}

In this section, we first show how to find an efficient collision path for SHAMATA. Recall from Sect. 3.4 that the new message freedom in each round of SHAMATA allows an adversary to linearise the $A R F^{r}$ function in one of the two clockings in a round. Thus, we aim to find a collision differential path that activates the $A R F^{r}$ function in at most one clocking of each round as well. However, it was already pointed out in Sect. 3.4 that it is impossible to avoid this in the round where the first difference is introduced, but we can aim to avoid this in all the other rounds. We describe two methods to achieve this. The first method is based on searching low-weight codewords of a linear code and the second method is a simple exhaustive search. The former is more general and can also be used to find differential paths spanning a long message. The latter is only feasible for short messages, but it is simpler. In the case of SHAMATA, either of the methods can be used to achieve the same result. 


\subsection{Low-Weight Codewords}

For a fixed number of message blocks, all differential paths under consideration can be seen as the codewords of a linear code. We show that searching for lowweight codewords in this code is a useful tool to construct good differential paths for SHAMATA. The use of low-weight codeword search techniques to construct differential paths was proposed by Rijmen and Oswald 9] and extended by Pramstaller et al. in 8 .

A codeword of the code under consideration contains, for each round, the message difference and the differences in the internal state registers immediately after the new message block was added. As we consider only $\Delta$ differences, each of these differences is represented by a single bit. Let $\Delta m^{(i)}, \Delta b_{3}^{(i)}, \ldots, \Delta b_{0}^{(i)}$ and $\Delta k_{11}^{(i)}, \ldots, \Delta k_{0}^{(i)}$ denote these bits for round $i$. With $N$ the fixed number of message blocks used, a codeword of the code is then given by

$$
\left[\Delta m^{(1)} \cdots \Delta m^{(N)}\left\|\Delta b_{3}^{(1)} \cdots \Delta k_{0}^{(1)}\right\| \cdots \| \Delta b_{3}^{(N)} \cdots \Delta k_{0}^{(N)}\right] .
$$

We now construct the generator matrix $\mathbf{G}$ of this code. The differences in a SHAMATA state immediately after the message addition in round $i$ can be represented by an $1 \times 16$ binary vector $\Delta s^{(i)}$,

$$
\Delta s^{(i)}=\left[\Delta b_{3}^{(i)} \cdots b_{0}^{(i)} k_{11}^{(i)} \cdots k_{0}^{(i)}\right] .
$$

As the $A R F^{r}$ function is assumed to behave linearly with respect to the $\Delta$ difference, the state difference vector in round $i, \Delta s^{(i)}$, can be written in function of the state differences vector in round $i-1, \Delta s^{(i-1)}$, as follows

$$
\Delta s^{(i)}=\Delta s^{(i-1)} \cdot \mathbf{A} \oplus \Delta m^{(i)} \cdot w .
$$

Here, $w$ is a $1 \times 16$ vector indicating to which positions of the internal state a new message block is added. It is easy to see that

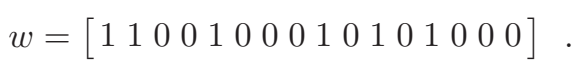

The $16 \times 16$ matrix $\mathbf{A}$ is a transition matrix corresponding to the two clockings in the round. It is given by

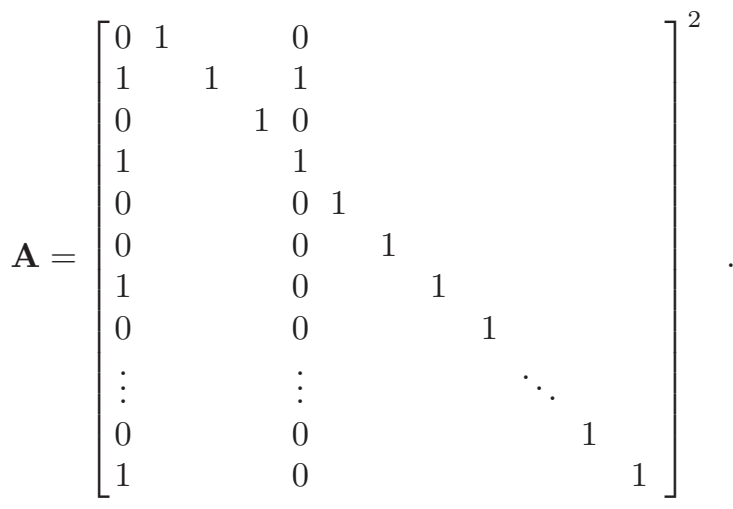


Now, consider the $N \times 17 N$ generator matrix $\mathbf{G}_{\text {all }}$ given by

$$
\mathbf{G}_{\text {all }}=\left[\begin{array}{ccccc}
w & w \mathbf{A} & w \mathbf{A}^{2} & \cdots & w \mathbf{A}^{N-1} \\
& w & w \mathbf{A} & \cdots & w \mathbf{A}^{N-2} \\
& & w & & \vdots \\
& & & \ddots & w \mathbf{A} \\
& & & & w
\end{array}\right] .
$$

This is the generator matrix of a linear code that contains all length $N$ differential paths of the type we consider. As we are only interested in collision differentials, it is required that the last internal state has no difference. This can be achieved by using Gaussian elimination to force zeroes in the last 16 columns of $\mathbf{G}_{\text {all }}$. This gives the generator matrix $\mathbf{G}$, which generates a linear code containing all differential paths that result in a collision.

Due to the possibility of message modification in either of the clockings in a SHAMATA round, but not both (see Sect. 3.4), a good differential path for SHAMATA activates the $A R F^{r}$ function in at most one clocking per round. As was already noted, it is impossible to avoid activating $A R F^{r}$ in both clockings of the round where a difference is first introduced. But we aim to avoid this in the remainder of the differential path.

Intuitively, a codeword with a low weight in $\Delta b_{2}$ and $\Delta b_{3}$, which are the input differences to $A R F^{r}$, is more likely to satisfy this property than a random codeword. Thus, we look for low-weight codewords in this code, considering only the weight of these bits, using an algorithm similar to that of Canteaut and Chabaud [3]. For each codeword below a certain threshold weight, we check if it satisfies the condition mentioned above. If it does, a suitable collision differential path has been found. If not, the search is simply continued. Note that this search method can find collision differential paths shorter than $N$ rounds. Indeed, nothing prevents the search from padding a shorter differential path to $N$ rounds by adding rounds without a difference, as we indeed observed. The shortest collision differential path we found is shown in Table 1, It consists of 25 rounds and, except for the first round, only activates $A R F^{r}$ in at most one of the clockings of a round.

\subsection{An Alternative Approach}

Note that, for a given length of $N$ rounds, there are only $2^{N}$ possible differential paths of the type we consider. Indeed, as each message block can only have a $\Delta$ difference or no difference at all, there are only $2^{N}$ possible message differences. Given the message difference, exactly one differential path follows. Hence, when $N$ is not too large, a simple brute force search can also be a viable approach.

As the more general approach given above resulted in a differential path of only 25 rounds, a brute force approach is indeed practically feasible. We have exhaustively searched all differential paths of length up to 25 rounds. As expected, this search also found the differential path given in Table 1. Moreover, there is only one differential path of 25 rounds, and no shorter differential paths of this type exist. Hence, the differential path in Table 1 is optimal. 
Table 1. The differential path for 25 rounds of SHAMATA with differences after each clocking. For differences at the input of $A R F^{r}$ (word $B_{1}$, grey column), the differential probabilities of each round are given in the last two columns for SHAMATA-256 $\left(A R F^{1}\right)$ and SHAMATA-512 $\left(A R F^{2}\right)$.

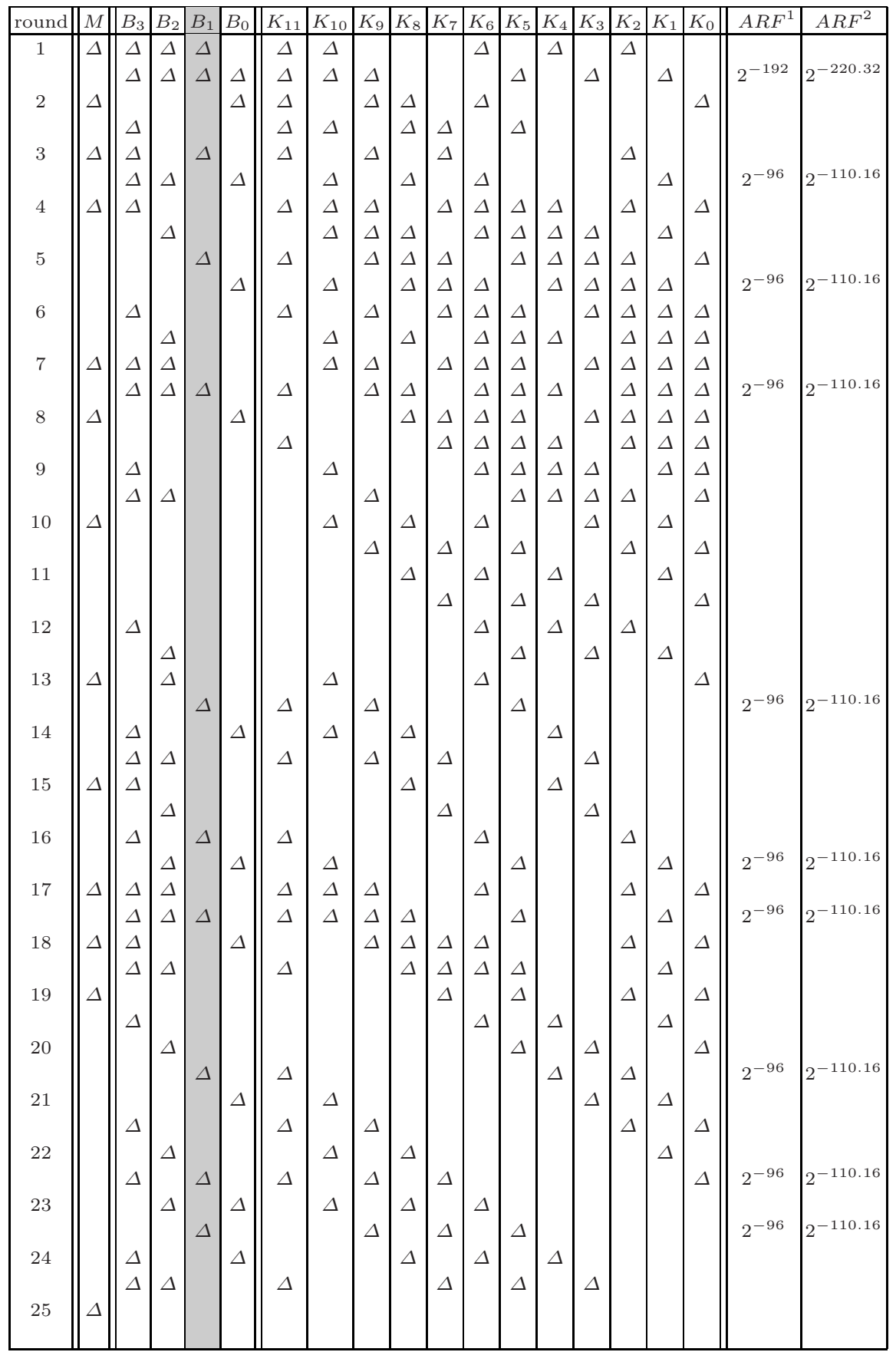




\section{Collision Attack on SHAMATA}

In this section, we put together the various pieces that were introduced, and present our collision attack on SHAMATA. We search for a message pair which follows the differential path in Table 1

\subsection{Collisions for SHAMATA-256 and SHAMATA-512}

In rounds where none of the $A R F^{r}$ functions is active, the differential path is always followed, regardless of the message block. Hence, in those rounds, we make an arbitrary choice for the message block. In rounds with exactly one active $A R F^{r}$ function, the message modification technique presented in Sect.3.4 is used to deterministically construct a message block that ensures that the differential path is followed. This takes only negligible time, i.e., no more than computing a single round of SHAMATA.

However, in the first round where a difference is introduced, the $A R F^{r}$ function is active in both clockings. The message modification technique of Sect. 3.4 can only deterministically satisfy the conditions for one of them. As discussed in Sect. 3.4 the probability that the path is still followed is $2^{-96}$ for $A R F^{1}$ (SHAMATA-256) and $2^{-110.16}$ for $A R F^{2}$ (SHAMATA-512). A prefix with no difference is used to provide the required message freedom.

Thus, a conforming pair for the first round of the differential path can be found by performing about $2^{96}$ trials for SHAMATA-256 and about $2^{110}$ trials for SHAMATA-512. Once such a pair has been found, a colliding message pair can be constructed with negligible additional effort. Thus, the overall complexity of our attack is about $2^{96}$ SHAMATA rounds for SHAMATA-256, and about $2^{110}$ SHAMATA rounds for SHAMATA-512. The attack requires only negligible memory and is easily parallelisable. Hence, for both variants of SHAMATA, the attack is significantly faster than a brute force attack. Note that the attack also applies to SHAMATA-224 and SHAMATA-384.

\subsection{Practical Collisions for SHAMATA-256}

In the case of SHAMATA-256, a more efficient approach exists to control the values which are input to the $A R F^{r}$ function in both clockings of a round. This approach exploits the fact that in SHAMATA-256 only a single AES round is used, i.e., $r=1$. Hence, this method can not be applied to SHAMATA-512, where $r=2$.

Assume we aim to fix the inputs to the $A R F^{1}$ function in both clockings of round $i$ to $X_{1}$ and $X_{2}$, respectively. Let $B^{(i)}$ denote the $B$-register at the beginning of round $i$. Then, this requirement can be written as

$$
\left\{\begin{array}{l}
B_{2}^{(i)} \oplus P^{(i)} \oplus i=X_{1} \\
B_{3}^{(i)} \oplus Q^{(i)} \oplus i=X_{2}
\end{array} .\right.
$$

Using the definition of the state update function of SHAMATA in (10)-(3), this can be rewritten in a function of the internal state at the beginning of round $i-1$ and the message blocks $M_{i-1}$ and $M_{i}$, yielding the following 


$$
\left\{\begin{array}{rl}
M_{i-1} & =M C^{-1}\left(D_{1} \oplus S B^{-1}\left(C_{1} \oplus S R^{-1}\left(M_{i}\right)\right)\right) \\
M_{i-1}^{\mathrm{T}} & =M C^{-1}\left(D_{2} \oplus S B^{-1}\left(C_{2} \oplus S R^{-1}\left(M_{i}^{\mathrm{T}}\right)\right)\right)
\end{array},\right.
$$

where $C_{1}, C_{2}, D_{1}$ and $D_{2}$ are constants defined by

$$
\begin{aligned}
& C_{1}=S R^{-1}\left(M C^{-1}\left(B_{0}^{(i-1)} \oplus K_{9}^{(i-1)} \oplus K_{0}^{(i-1)} \oplus i \oplus X_{1}\right)\right), \\
& C_{2}=S R^{-1}\left(M C^{-1}\left(B_{1}^{(i-1)} \oplus K_{10}^{(i-1)} \oplus K_{1}^{(i-1)} \oplus i \oplus X_{2}\right)\right), \\
& D_{1}=B_{2}^{(i-1)} \oplus(i-1), \\
& D_{2}=B_{3}^{(i-1)} \oplus(i-1) .
\end{aligned}
$$

These constants only depend on the internal state of SHAMATA-256 at the beginning of round $i-1$, and are thus known. Now, we search for message blocks $M_{i-1}$ and $M_{i}$ such that the conditions of (16) are satisfied.

A straightforward approach to find the message blocks $M_{i-1}$ and $M_{i}$ would be to guess one of them, compute the other using the first equation of (16) and then, check if the second equation of (16) holds as well. This procedure is expected to find a solution after about $2^{128}$ trials. We propose a guess-and-determine approach which performs significantly better. Our approach is as follows

1. Assume we know the four bytes of $M_{i}$ indicated in the pattern in Fig. 2(a). Note that this pattern is symmetric, i.e., it is invariant under matrix transposition. This implies that also the same pattern of bytes of $M_{i}^{\mathrm{T}}$ is known. Note that in (16), $M_{i}$ and $M_{i}^{\mathrm{T}}$ are input to the inverse ShiftRows operation or $S R^{-1}$. This operation performs a circular right shift of the rows of the state over $0,1,2$ or 3 bytes for the first, second, third and fourth row, respectively. Hence, the bytes of $M_{i}$ indicated in Fig 2 (a) form the first column of $S R^{-1}\left(M_{i}\right)$. Similarly, the first column of $S R^{-1}\left(M_{i}^{\mathrm{T}}\right)$ is known. All other operations in (16) treat the four columns independently, so knowledge of the first columns of $S R^{-1}\left(M_{i}\right)$ and $S R^{-1}\left(M_{i}^{\mathrm{T}}\right)$ suffices to compute the first columns of $M_{i-1}$ and $M_{i-1}{ }^{\mathrm{T}}$. The latter is equal to the first row of $M_{i-1}$, which overlaps with the first column of $M_{i-1}$ in exactly one byte.

Thus, we investigate all $2^{32}$ guesses for four bytes of $M_{i}$ as indicated in Fig. 2(a). For each guess, we compute the first column and the first row of $M_{i-1}$ using (16). Then, we verify if the overlapping byte matches, and if so, we save the candidate in a list $L_{1}$. As this imposes an 8-bit condition, about $2^{24}$ candidates are expected to remain.

2. The same procedure is repeated with the patterns in Fig.2(b), Fig.2(c) and Fig. 2(d). Each pattern is invariant under matrix transposition, and results in one column after applying the $S R^{-1}$ operation. This results in four lists, $L_{1}, L_{2}, L_{3}$ and $L_{4}$ of about $2^{24}$ elements each.

3. An element of the list $L_{1}$ contains candidate values of the first row and column of $M_{i-1}$. Similarly, an element of the list $L_{2}$ contains the second row and column of $M_{i-1}$. Note that these overlap in two byte positions. Thus, we can merge both lists and store all matching combinations in a new list, $L_{A}$. The expected number of entries in the new list $L_{A}$ is $2^{24} \times 2^{24} \times 2^{-16}=2^{32}$. 


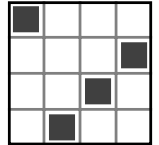

(a)

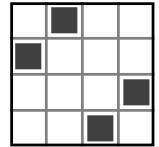

(b)

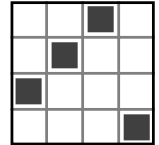

(c)

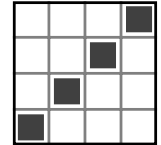

(d)

Fig. 2. Patterns used in the guess-and-determine phase

If the lists $L_{1}$ and $L_{2}$ are sorted according to the overlapping bytes, this merge operation can be performed very efficiently.

4. The same procedure is used to merge the lists $L_{3}$ and $L_{4}$, resulting in a new list $L_{B}$ which is also expected to contain about $2^{32}$ entries.

5. Finally, the lists $L_{A}$ and $L_{B}$ are merged. The entries in these lists overlap in eight byte positions, which corresponds to a 64-bit condition. Again, if both lists are sorted according to these bytes, merging them can be done efficiently. The number of expected matches is $2^{32} \times 2^{32} \times 2^{-64}=1$.

It is easy to verify that each final match will satisfy (16), and also that every solution to (16) will be found by this procedure. The time complexity of this algorithm is dominated by the merging of lists $L_{A}$ and $L_{B}$, which takes $2^{32}$ operations. Using hash tables as the data structure to store the lists, an explicit sorting step can be avoided. The memory complexity is determined by one of the lists $L_{A}$ or $L_{B}$, as only one of them really needs to be stored in memory, while the elements of the other can be computed on-the-fly. This corresponds to a memory requirement of about $2^{32}$ AES states.

For a practical implementation, it is better to reduce the memory requirements of the algorithm, at the expense of an increase in its time complexity. This can be done by, for instance, fixing the byte in the first row and last column of $M_{i-1}$ a priori. Then, the lists $L_{1}$ and $L_{4}$ are only expected to contain $2^{16}$ elements each, and the lists $L_{A}$ and $L_{B}$ are reduced to about $2^{24}$ elements. Thus, the total memory complexity is reduced to about $2^{24}$ AES states, or $256 \mathrm{MB}$. However, as one byte was fixed a priori, the entire procedure has to be repeated $2^{8}$ times, increasing the time complexity to $2^{40}$ operations. We have implemented our attack. The guess-and-determine phase was run on a cluster using 256 jobs with a running time of about 5 minutes each. The rest of the attack takes only negligible time using message modification, as explained in Sect. 3.4. A collision example for SHAMATA-256 is given in App. A.

\section{Conclusion}

In this paper, we have presented a practical collision attack on the SHA-3 submission SHAMATA. Due to weaknesses in the message injection and state update function of SHAMATA it is possible to find certain message differences, that do not get changed by the message expansion or the non-linear part of the state 
update function. These symmetric XOR differences need to be equal in each byte of the 128-bit words. Using these differences, the non-linear $A R F^{r}$ function behaves linearly and we can search for a differential path using a linearised variant of SHAMATA. Moreover, since we use the same difference in every 128-bit word, we can represent each word of the internal state by a single bit.

The main weakness in SHAMATA is the relatively light message injection followed by a low number of register clockings. The message injection allows us to efficiently fulfil many conditions using basic message modification. This results in an attack complexity of about $2^{96}$ for SHAMATA-256 and $2^{110}$ for SHAMATA512. Using an efficient guess-and-determine technique we are able to improve the complexity of the attack on SHAMATA-256 to about $2^{40}$ round computations and present a practical collision for SHAMATA-256. Possible improvements for SHAMATA include increasing the number of times the internal registers are clocked and the use of constants to avoid the use of symmetric differences.

\section{Acknowledgements}

This work was supported in part by the IAP Programme P6/26 BCRYPT of the Belgian State (Belgian Science Policy), and in part by the European Commission through the ICT programme under contract ICT-2007-216676 ECRYPT II. The collision example for SHAMATA-256 was obtained utilizing high performance computational resources provided by the University of Leuven, http:// ludit.kuleuven. be/hpc.

\section{References}

1. Atalay, A., Kara, O., Karakoç, F., Manap, C.: SHAMATA Hash Function Algorithm Specifications. Submission to NIST (2008),

http://www. uekae.tubitak.gov.tr/uekae_content_files/crypto/

SHAMATASpecification.pdf, http://www . uekae. tubitak.gov . tr/home.do?ot=1\&sid=601\&pid=547

2. Bertoni, G., Daemen, J., Peeters, M., Assche, G.V.: Sponge functions. In: ECRYPT Hash Workshop, Barcelona, Spain, May 24-25 (2007), http://sponge. noekeon.org/SpongeFunctions.pdf

3. Canteaut, A., Chabaud, F.: A New Algorithm for Finding Minimum-Weight Words in a Linear Code: Application to McEliece's Cryptosystem and to Narrow-Sense BCH Codes of Length 511. IEEE Transactions on Information Theory 44(1), 367378 (1998)

4. Daemen, J., Assche, G.V.: Producing Collisions for Panama, Instantaneously. In: Biryukov, A. (ed.) FSE 2007. LNCS, vol. 4593, pp. 1-18. Springer, Heidelberg (2007)

5. Daemen, J., Clapp, C.S.K.: Fast Hashing and Stream Encryption with PANAMA. In: Vaudenay, S. (ed.) FSE 1998. LNCS, vol. 1372, pp. 60-74. Springer, Heidelberg (1998)

6. Daemen, J., Rijmen, V.: The Design of Rijndael: AES — The Advanced Encryption Standard. Springer, Heidelberg (2002) 
7. National Institute of Standards and Technology: Announcing Request for Candidate Algorithm Nominations for a New Cryptographic Hash Algorithm (SHA-3) Family. Federal Register 27(212), 62212-62220 (November 2007), http://csrc.nist.gov/groups/ST/hash/documents/FR_Notice_Nov07.pdf

8. Pramstaller, N., Rechberger, C., Rijmen, V.: Exploiting Coding Theory for Collision Attacks on SHA-1. In: Smart, N.P. (ed.) Cryptography and Coding 2005. LNCS, vol. 3796, pp. 78-95. Springer, Heidelberg (2005)

9. Rijmen, V., Oswald, E.: Update on SHA-1. In: Menezes, A. (ed.) CT-RSA 2005. LNCS, vol. 3376, pp. 58-71. Springer, Heidelberg (2005)

10. Rijmen, V., Van Rompay, B., Preneel, B., Vandewalle, J.: Producing Collisions for PANAMA. In: Matsui, M. (ed.) FSE 2001. LNCS, vol. 2355, pp. 37-51. Springer, Heidelberg (2002) 


\section{A Colliding Message Pair for SHAMATA-256}

$\mathrm{m} 1=$

00000000: $1037 \mathrm{fd} \mathrm{e} 76530$ 1c c0 e3 61 6e $41246 \mathrm{f}$ cb b9 |.7..e0...anA\$o..। 00000010: 7f $288117814 \mathrm{a}$ d1 $3 f$ bf 4 e ca da 92 f5 35 do |.(...J.?.N...5.। 00000020: f0 f0 dc 1973 d5 a7 078 ob bc 3d b6 $854657 \quad|\ldots . . . \ldots . . . . F W|$ 00000030: 0292 d1 2400 df 4067 ca 2c fa 5b $9 d 70$ 2c ce |..\$..@g.,.[.p,.। 00000040: de 3851 f5 $013 c$ bb aa d8 ba 38 0e a1 40 b1 91 |.8Q..<;..8....। 00000050: 7b 181824 cc d9 76 c0 f7 4a 6128860630 e $\mid\{\ldots \$$....Ja(..0.| 00000060: $308 \mathrm{~d}$ ab a3 6252 aa ee $5 \mathrm{~d} 66$ 2b 13 ec $716 \mathrm{~b}$ ca $10 \ldots . . b R ..] f+\ldots q k . \mid$ 00000070: e3 29 f2 2 c b3 ed $3 d 7$ e f7 f2 fd ob 1 e c7 d6 e5 |.)....= ...... 00000080: aa bc bf ab f9 fb 56 d1 b5 8 e df 57 ce 90 e8 fe |............. 00000090: 1e 93 a2 80 e6 4c 6f 43 b3 9a 57 9f 0c c2 69 b6 |....LoC..W...i.। 000000a0: 7e 29617724 b7 48 d9 45273013 b8 1912 d6 | ) aw\$.H.E’0....। 000000b0: ac b4 569200 c5 d6 b3 60 2d 52 6c ef bc 22 6d I..V....'-RI.."ml 000000c0: e5 83 e5 09 3b 2d e2 80551394 0d 2c a6 e3 d8 $|\ldots \ldots ;-\ldots . \ldots, \ldots|$ 000000d0: 53 e9 016672 ae $8 d$ cf $68258 a$ b6 ae 64 e7 c1 IS..fr...h\%...d..। 000000e0: $5 \mathrm{a} 39 \mathrm{6b} 5 \mathrm{a}$ ff 41 ee 5 f 6 e $60 \mathrm{cb} 5 \mathrm{~d} 1 \mathrm{c}$ ed ca 01 |Z9kZ.A._n'.]...। 000000fo: 70 af $0 \mathrm{a}$ ab dd ed 2c 3200 c0 3f 2c 662204 c0 lp....,2..?,f"..। 00000100: 3b 9765 9d 016498 7b e6 63 d4 d6 4b 7700 bb l;.e..d.\{.c..Kw..। 00000110: bb ac 35 e3 27665534 oc of db d7 2 f 1619 ae $\mid . .5$.'fU4......। 00000120: 5b 6f 1a 5a b0 28 b9 1e 8984 7b a5 7146 a7 e2 |[o.Z.(...\{.qF..।

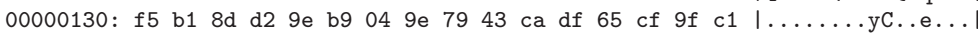
00000140: bb f6 43 f9 cd 88 af 13 ea 2 f 93 e8 cd 39 8c a0 ।...........। 00000150: 3e ba $1 \mathrm{~b}$ ef e2 d5 0d $6 \mathrm{~b} 598911 \mathrm{cb}$ cf b8 ad c4 $\mid>\ldots \ldots k Y \ldots \ldots$. 00000160: 1a 3f 2 f 9d a3 1d 82 3c e0 75 gd 83 b2 ac 3c bf $|. ? / \ldots .<. \ldots<$. 00000170: e0 27 0c c5 af b0 be a9 94 1e de 9 d 506910 cb |.'.......Pi..। 00000180: 69 3a 9708 f4 9b a6 6d df 71 4d 4440 ec 057 e |i:....m.qMDQ.. । 00000190: a6 21 6d 89 f6 7b f4 4 f 0405 1a d3 bd c7 9727 |.!m..\{.0....'।

SHAMATA-256 $(\mathrm{m} 1)=$

00000000: 6e a3 b1 a1 2975 dd $3 f$ f5 60 f8 1 b 6 b 1102 9a $\mid n . \ldots) u . ? . ' \ldots k . . \mid$ 00000010: 14 b9 b2 d9 b3 2a b6 02 2a f5 83 ab e3 4c 1a 2a |.............|

$\mathrm{m} 2=$

00000000: $1037 \mathrm{fd} \mathrm{e} 76530$ 1c c0 e3 61 6e $41246 \mathrm{f}$ cb b9 |.7..e0...anA\$o..।

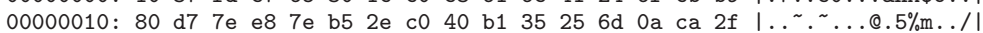
00000020: Of of 23 e6 8c 2 a 58 f8 73 f4 43 c2 49 7a b9 a8 ।..\#...X.s.C.Iz..। 00000030: fd $6 \mathrm{~d} 2 \mathrm{e} \mathrm{db}$ ff 20 bf 9835 d3 05 a4 628 f d3 31 ।.m..........1। 00000040: 21 c7 ae 0a fe c3 c4 552745 c7 f1 5e bf 4 e 6 e |!......'E.. ..Nn| 00000050: 7b 181824 cc d9 76 c0 f7 4a 6128860630 e |\{..\$....Ja(..0.| 00000060: 30 8d ab a3 6252 aa ee $5 d 66$ 2b 13 ec $716 b$ ca $10 \ldots . . b R ..] f+\ldots q k . \mid$ 00000070: 1c d6 0d d3 4c 12 c2 8108 0d 02 f4 e1 38 29 1 a |.........8).।

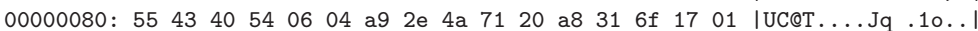
00000090: 1e 93 a2 80 e6 4c $6 f 43$ b3 9a 57 9f 0c c2 69 b6 ।....LoC..W...i.। 000000a0: 81 d6 9 e 88 db 48 b7 26 ba d8 cf ec 47 e6 ed 29 |............)। 000000b0: ac b4 569200 c5 d6 b3 60 2d 52 6c ef bc $226 d$ ।..V....'-RI.."ml 000000c0: e5 83 e5 09 3b 2d e2 80551394 0d 2c a6 e3 d8 $|\ldots \ldots ;-\ldots . \ldots, \ldots|$ 000000d0: ac 16 fe 998 d 51723097 da $7549519 b 18$ 3e |....Qr0..uIQ..>। 000000e0: $5 \mathrm{a} 39 \mathrm{6b} 5 \mathrm{a}$ ff 41 0e 5 f 6 e $60 \mathrm{cb} 5 \mathrm{~d} 1 \mathrm{c}$ ed ca $01 \mid \mathrm{Z} 9 \mathrm{kZ} . \mathrm{A}_{\text {._n }}$ '.].... 000000f0: 8f 50 f5 542212 d3 cd ff $3 f$ c0 d3 99 dd fb 3f |.P.T"...?...?। 00000100: 3b 9765 9d $0164987 \mathrm{~b}$ e6 63 d4 d6 4 b 7700 bb |;.e..d.\{.c..Kw..। 00000110: $4453 \mathrm{ca} 1 \mathrm{c}$ d8 99 aa cb f3 f0 2428 d0 e9 e6 51 |DS.....\$(...Q| 00000120: a4 90 e5 a5 4 f d7 46 e1 76 7b 84 5a 8 e b9 58 1d |...0.F.v\{.Z..X.। 00000130: 0a 4e 72 2d $6146 \mathrm{fb} 6186$ bc $35209 \mathrm{a} 3060$ 3e $\left|. N r-a F . a .5 \quad .0^{\prime}>\right|$ 00000140: bb f6 43 f9 cd 88 af 13 ea 2 f 93 e8 cd 39 8c a0 |...........। 00000150: 3e ba $1 \mathrm{~b}$ ef e2 d5 0d $6 \mathrm{~b} 598911 \mathrm{cb}$ cf b8 ad c4 $|>\ldots \ldots k Y \ldots \ldots|$ 00000160: 1a 3f 2 f 9d a3 1d 82 3c e0 75 9d 83 b2 ac 3c bf $|. ? / \ldots . . . .<$. 00000170: e0 27 Oc c5 af b0 be a9 94 1e de 9 d $506910 \mathrm{cb} \mid ., \ldots \ldots . . . . \mathrm{Pi}$ 00000180: 69 3a 9708 f4 9b a6 6 d df 71 4d 4440 ec 057 e |i:....m.qMD@.. । 00000190: 59 de 92760984 ob b0 fb fa e5 2c 423868 d8 |Y..v.....,B8h.|

SHAMATA-256 $(\mathrm{m} 2)=$

00000000: 6e a3 b1 a1 2975 8d $3 f$ f5 60 f8 1 b 6 b 1102 9a $\mid \mathrm{n} . \ldots)$ u.?.'.k...। 00000010: 14 b9 b2 d9 b3 2a b6 02 2a f5 83 ab e3 4c 1a 2 a |...........*। 